\title{
Prevalence of late stage in 507 Medication-related osteonecrosis of the jaws (MRONJ) sites: comparison between cancer and osteometabolic patients
}

\author{
Giulia Ghidini ${ }^{1}$, Pierpaolo De Francesco ${ }^{1}$, Marco Frontera ${ }^{1}$, Maddalena Manfredi ${ }^{1}$, Marco Meleti ${ }^{1}$, paolo vescovi \\ 1 University of Parma
}

Funding: The author(s) received no specific funding for this work.

Potential competing interests: The author(s) declared that no potential competing interests exist.

\section{Abstract}

The present retrospective study we compared 507 sites of MRONJ occurred in 394 patients to highlight possible differences between cancer and non-cancer patients with stage III MRONJ.

Five hundred and seven sites of MRONJ were observed between January 2004 and March 2021 at the Center of Oral Medicine and Laser Surgery of the Academic Hospital of University of Parma. Patients were sub-classified in two groups: Group A (cancer) consisted in 293 affected patients and Group B (non-cancer) in 100 patients with MRONJ. Both Group A and B were evaluated on: gender, mean age, underlying pathology and MRONJ stage.

This retrospective analysis highlighted no main differences between cancer and non-cancer patients with stage III MRONJ: both groups presented with similar percentage of advanced stage of MRONJ.

Contrary to general belief: osteometabolic patients are not to be considered "safe" from serious complications rather than cancer patients.

\section{Introduction}

In advanced stages of MRONJ complications as pathologic mandibular fracture, involvement of the maxillary sinus and extra-oral fistula are possible serious complications radically worsening patients quality of life. ${ }^{[1][2]}$

In order to improve prevention of this impairing side effect, the analysis of the epidemiological aspect in affected patients has shown to be useful to better target screening strategies, aiming to early diagnosis. ${ }^{[3]}$

In the present retrospective study we compared 507 sites of MRONJ occurred in 394 patients to highlight possible differences between cancer and non-cancer patients with stage III MRONJ.

Materials and Methods 
Five hundred and seven sites of MRONJ were observed between January 2004 and March 2021 at the Center of Oral Medicine and Laser Surgery of the Academic Hospital of University of Parma. Patients were sub-classified in two groups: Group A (cancer) consisted in 293 affected patients and Group B (non-cancer) in 100 patients with MRONJ. Both Group A and B were evaluated on: gender, mean age, underlying pathology and MRONJ stage.

\section{Results}

Three hundred ninety-two MRONJ occurred in 293 cancer patients and 115 MRONJ occurred in 100 non-cancer patients. Group A consisted of 150 (38.2\%) Male and 242 Female (61,8\%) mean age 67 (MAX 88 - min 35), while B consisted of 8 Male (6.9\%) and 108 Female (93.1\%) mean age 74 (MAX 92- min 48).

In Group A the majority of patients were treated for for breast cancer, 166 (42.3\%), 122 for multiple myeloma (31.3\%) and $48(12.2 \%)$ for prostatic cancer.

In Group B 96 patients were treated for osteoporosis (83.5\%), 8 (6.9\%) for rheumatoid arthritis, 10 (8.7\%) for both osteoporosis and rheumatoid arthritis and one patient was treated for osteoporosis and sclerodermia (0.9\%). Stage III was diagnosed in 60 (15.3\%) patients in Group A and 18 (15.6\%) in Group B.

In Group A: 33 patients presented with sinusitis (55\%), 18 (30\%) with extra oral fistula and 6 (10\%) with mandibular fracture. Two patients (3.3\%) presented with a combination of fistula and fracture while one (1.7\%) had involvement of the maxillary sinus and extra oral fistula.

In Group B: the majority of patients showed involvement of the maxillary sinus (14, $77.8 \%), 2$ patients (11\%) presented with mandibular fracture and 1 (5.6\%) with fistula. One patient presented with both extra oral fistula and mandibular fracture.

\section{Conclusion}

This retrospective analysis highlighted no main differences between cancer and non-cancer patients with stage III MRONJ: both groups presented with similar percentage of advanced stage of MRONJ.

Management of stage III MRONJ is difficult and requires a non-conservative treatment in patients often with poor general health conditions.

As shown by published literature: the early diagnosis of MRONJ allows conservative approaches and therefore, prevention of complications can benefit from early detection and treatment of MRONJ at initial stages (stage I) in order to stabilize the disease and limit its evolution. ${ }^{[4][5][6]}$

Contrary to general belief: osteometabolic patients are not to be considered "safe" from serious complications rather than cancer patients.

Finally, since stage III concern both cancer and non-cancer patients equally, high attention must be paid to oncological 
patients but also to the wide numbers of patients treated with bisphosphonates, anti-resorptive and anti-angiogenic monoclonal antibodies for osteometabolic disorders.

\section{References}

1. 'Salvatore L. Ruggiero, Thomas B. Dodson, John Fantasia, Reginald Goodday, et al. (2014). American Association of Oral and Maxillofacial Surgeons Position Paper on Medication-Related Osteonecrosis of the Jaw-2014 Update. Journal of Oral and Maxillofacial Surgery, vol. 72 (10), 1938-1956. doi:10.1016/j.joms.2014.04.031.

2. 'Giuseppina Campisi, Rodolfo Mauceri, Francesco Bertoldo, Giordana Bettini, et al. (2020). Medication-Related Osteonecrosis of Jaws (MRONJ) Prevention and Diagnosis: Italian Consensus Update 2020. IJERPH, vol. 17 (16), 5998. doi:10.3390/ijerph17165998.

3. 'Giulia Ghidini et al.. (2017). Medication-related osteonecrosis of the jaw: risk factors in patients under biphosphonate versus patients under antiresorptive-antiangiogenic drugs. Minerva Stomatologica, vol. 66(4):135-140 .

4. 'Oliver Ristow, Thomas Rückschloß, Michael Müller, Moritz Berger, et al. (2019). Is the conservative non-surgical management of medication-related osteonecrosis of the jaw an appropriate treatment option for early stages? A longterm single-center cohort study. Journal of Cranio-Maxillofacial Surgery, vol. 47 (3), 491-499. doi:10.1016/j.jcms.2018.12.014.

5. ^Aliya A Khan, Archie Morrison, David A Hanley, Dieter Felsenberg, et al. (2014). Diagnosis and Management of Osteonecrosis of the Jaw: A Systematic Review and International Consensus. J Bone Miner Res, vol. 30 (1), 3-23. doi:10.1002/jbmr.2405.

6. 'Vescovi P et al. (2010). Bisphosphonate-Related Osteonecrosis of the Jaw (BRONJ) therapy. A critical review. Minerva Stomatologica, vol. 59(4):181-213 . 